\title{
A case study for a new metrics for economic complexity: The Netherlands
}

\author{
Andrea Zaccaria • Matthieu Cristelli • \\ Roland Kupers • Andrea Tacchella • \\ Luciano Pietronero
}

Received: 19 February 2014 / Accepted: 5 January 2015 / Published online: 29 January 2015

C The Author(s) 2015. This article is published with open access at Springerlink.com

\begin{abstract}
We present a new approach for the economic analysis of countries, which we apply to the case of the Netherlands. Our study is based on a novel way to quantify exported products' complexity and countries' fitness which has been recently introduced in the literature. Adopting a framework in which products are clustered in sectors, we compare the different branches of the export of the Netherlands, taking into account the time evolution of their volumes, complexities and competitivenesses in the years 1995-2010. The High Tech and Life Sciences sectors share high quality products but low competitiveness; the opposite is true for Horticulture and Energy. We analyze in detail the Chemicals sector, finding a declining global complexity which is mostly driven by a shift towards products of lower quality. A growth forecast is also provided. In light of our results we suggest a differentiation in policy between the country's self-defined industrial sectors.
\end{abstract}

Keywords Complexity $\cdot$ Competitiveness $\cdot$ Trade $\cdot$ Industry

JEL Classification $\mathrm{C} 6 \cdot 011 \cdot \mathrm{C} 23$

A. Zaccaria $(\varangle) \cdot$ M. Cristelli $\cdot$ A.Tacchella $\cdot$ L. Pietronero

ISC-CNR, via dei Taurini, 19, 00185 Rome, Italy

e-mail: and.zaccaria@gmail.com

R. Kupers

Smith School of Enterprise and the Environment, University of Oxford, Hayes House,

75 George Street, Oxford, UK

A. Tacchella $\cdot$ L. Pietronero

Dip. di Fisica, "Sapienza" University of Rome, P.le A. Moro 2, 00185 Rome, Italy 


\section{Introduction}

One of the most important fields of research in Economics concerns the study of economic growth and the analysis of the causes leading to the observed differences in growth rates across countries. In the standard economic literature one usually considers the so called production functions, which connect the observed or predicted growth to suitable proxies for human and physical capital (Krugman and Wells 2013). However, when the correlation between the growth rate and various state variables is studied, mixed results are found (Barro and Sala-i Martin 2004). For example, the presence of a connection between growth and investment spendings in Research and Development has been questioned on an empirical basis by Jones (1995). Hausmann and Rodrick have proposed a general equilibrium model in which a central role is played by the learning process of countries, and in particular by their ability to identify which technologies can be better adapted to their situations (Hausmann and Rodrick 2003).

Recently, an approach based on the Science of Complexity (Anderson 1972) have been proposed by Hidalgo and Hausmann (2009). These authors claim that their Economic Complexity Index outperforms various explanatory variables which have been proposed in the economic literature, such as the Worldwide Governance Indicators calculated by the World Bank, the World Economic Forum's Global Competitiveness Index, and various educational measures (see Hausmann et al. 2011 for a detailed analysis). Hidalgo and Hausmann's approach has been criticized and deeply revisited in Tacchella et al. (2012), Caldarelli et al. (2012), Tacchella et al. (2013), Cristelli et al. (2013). The analyses of the present paper are based on this last approach, which is founded on a new viewpoint on the relation between the countries and the products they export. The standard economic approach (Smith 1776; Ricardo 1817; Romer 1990; Grossman and Helpman 1991; Flam and Flanders 1991) to analyze the exports of a country relies on the assumption that the best strategy is to focus on a few, firstrate products. These products would capture a consistent fraction of the available market, assuring high profits and employment. In order to decide in which products a country should specialize, Ricardo utilized the concept of comparative advantage, showing that if countries produce and trade only some chosen products the system will obtain a total improvement. A contrary view, explained for example in Dosi et al. (1990), suggests instead that countries focus on those products in which they have an absolute advantage, which relies mainly on technology. ${ }^{1}$ In order to explain the empirical evidence for countries' diversification one can single out the new trade theory (Krugman 1979), in which a production function of the form proposed in Dixit and Stiglitz (1977) is used. However, as correctly pointed out by Hidalgo and Hausmann (2010), such an approach does not give, in principle, any clue about which products will be chosen by countries, and in particular by the less diversified ones. In order to study this fundamental problem, both combinatorial (Hidalgo and Hausmann 2010) and network-based approaches has been proposed (Hidalgo et al. 2007; Zaccaria et al. 2014).

\footnotetext{
1 We have checked that the main features of our analysis, which rely on the triangular structure of the export matrix, are not much dependent on the choice of the specific measure to assess countries' export baskets.
} 
Empirically, the structure of the country-product matrix is peculiar and suggests that the assumption of countries' specialization is not valid. Let us consider the matrix $M_{c p}$ whose entries take the value 1 if the country $c$ exports the product $p$ and 0 otherwise. In practice, we computed the Revealed Comparative Advantage as defined by Balassa (1965), ${ }^{2}$ and we used a threshold value of 1 to make the matrix binary, as done in Hidalgo and Hausmann (2009) and Tacchella et al. (2012). Let us arrange rows from the most to the less diversified country and columns from the most to the less ubiquitous product. In a Ricardian world we would expect to find a block diagonal matrix in which each country is specialized in a small set of suitable products. On the contrary, as shown in Hidalgo and Hausmann (2009), Tacchella et al. (2012), Caldarelli et al. (2012), $M_{c p}$ turns to be triangular. In particular, the countries which export rare and complex products are, on a first approximation, the ones that we would call developed, at least from the point of view of the usually adopted indicators; the important point is that these countries are also the most diversified ones. This observation leads to the introduction of a new metrics for products' complexity and countries' fitness (Tacchella et al. 2012), which is particularly suitable to study the country's competitiveness with respect to the global market. This methodology will be explained in a more detailed way in the next section. ${ }^{3}$ In this paper we use this metrics to study the characteristics of one country in particular, the Netherlands. We believe that this country, among the many that could have been chosen to illustrate this methodology, can be an interesting test bed for the following reasons: i) The Netherlands has a well diversified export basket; as a consequence, a broad range of products can be included in our analysis. ii) This country has articulated an explicit policy to build economic advantage based on groupings of products and industries into sectors. This raises the question whether our methodology enables a test of the relative competitiveness of such self-defined sectors for industrial policy. We find that it does so, although to the exclusion of services sectors with are not covered by the methodology. iii) The Netherlands did not experience political and social turmoil, which may, a priori, undermine an export based analysis. iv) Not least, one of the authors (RK) has a relevant experience with respect to this specific country, among many others, and in particular he has been involved in consulting about strategic planning and industrial development.

The paper is organized as follows. First we introduce the methodology to measure our quantities of interest, namely countries' fitness and products' complexity, starting from the yearly export flows. Then we describe our data and we illustrate the principle of the products' division in sectors which we will adopt in the rest of the paper. At this point we analyze the exports of the Netherlands using the previously introduced framework, with a particular emphasis on the time evolution of volumes and complexities, the export spectroscopy and a detailed analysis of a sample sector, Chemicals.

\footnotetext{
${ }^{2}$ For each country and product, the Revealed Comparative Advantage is the ratio of two ratios: the numerator is the weight of the product with respect to that country's basket; the denominator is the weight of the total trade of that product with respect to the total world export. This normalization naturally takes into account the country's size.

3 We point out that this coupling between countries' and products' evaluations is a fundamental difference between this method and other measures of products' sophistication, as the one proposed by Lall (2006), which is based on exporters' income.
} 
The last section draws some conclusions and illustrates some perspectives for future work.

\section{A new metrics for countries' fitness and products' complexity}

In this section we illustrate a new approach to measure the fitness of countries and the complexity of the exported products. This methodology has been recently introduced by Tacchella et al. (2012). The idea is the following. Each country has some capabilities, which represent its social, cultural and technological structure [this concept has been discussed in the economic growth framework in Dosi et al. (1990) and Lall et al. (2006)]. The capabilities permit to produce and export products, so the extension of the distribution of products and their complexity are linked to each country fitness; in particular, the complexity of a product increases with the capabilities which are needed in order to produce it, and the fitness is a measure of the complexity and the diversification of the exported products. ${ }^{4}$ In order to make this line of reasoning more quantitative, the starting point is the global structure of the previously defined matrix $M_{c p}$. Once countries and products are suitably arranged, this matrix is triangular, showing that developed countries have a diversified export, while poor or less developed countries export fewer, lower complexity products. This is clear from Fig. 1, in which we show a spectroscopy ${ }^{5}$ of the Netherlands and three other countries. To obtain these figures we sort the products by complexity and we plot their volume. We have grouped the products into bins of 5 products each on a complexity basis for clarity purposes; we point out that the aim of this representation is only to have a single comprehensive view of the export quality and diversification, and not to analyze economic sectors, which is done in the following sections. One can see that the Netherlands cover a wide range of products and it is not focused on high nor low complexity products. This is what we expect from a high-fitness country; in fact, its position in the fitness ranking is 11. Also Japan has an excellent diversification, with a special focus on high complexity products, while Nigeria and Qatar export only products whose complexity is quite low, for example petroleum or simple by-products. It is interesting to notice that Qatar has one of the highest per capita GDPs: this fact is not immediately translated into a high number of capabilities. Diversification may lead to an immediate, zero-order estimate for countries' fitness as the number of products they export. A natural further step is to calculate the Fitness of a country as the sum of the complexities of the products it exports. The evaluation of products' complexity is more subtle. We start noticing that the fact that a developed country exports a given product gives little information on the complexity of that product, because developed countries are diversified and so they export products with different qualities. On the contrary, if a product is exported by a country with a low fitness score, we will know that it can not be too complex. As a consequence, one has to assign complexities in such a way to weight more the

\footnotetext{
${ }^{4}$ In the following, we will use the export as a proxy for production. We are aware of the roughness of this approximation. However, we do not have access to similar databases on internal markets, and we believe that this partial analysis can contribute to the study of countries' development and growth in a significant way.

5 In Physics the spectroscopy is the quantification of the constituents of a physical or chemical compound.
} 
Netherlands

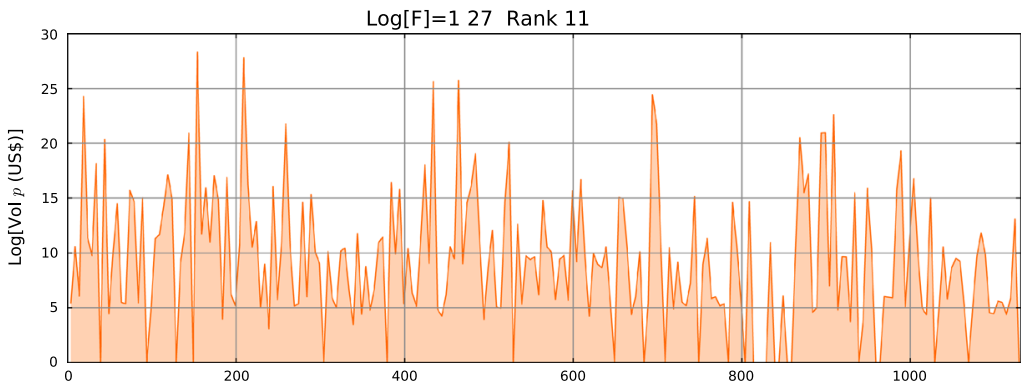

Japan

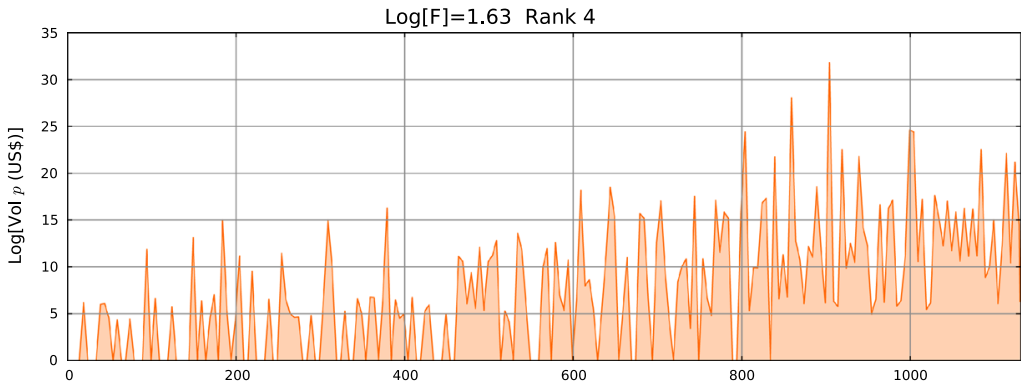

Nigeria

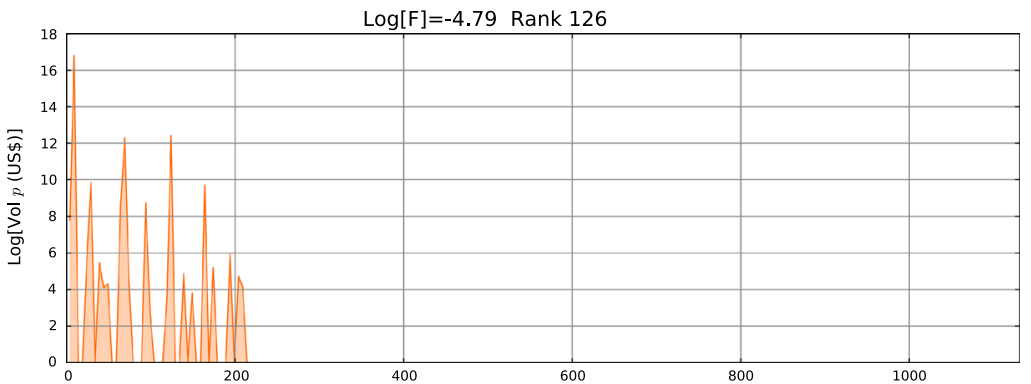

Qatar

$\log [\mathrm{F}]=-8.07$ Rank 142

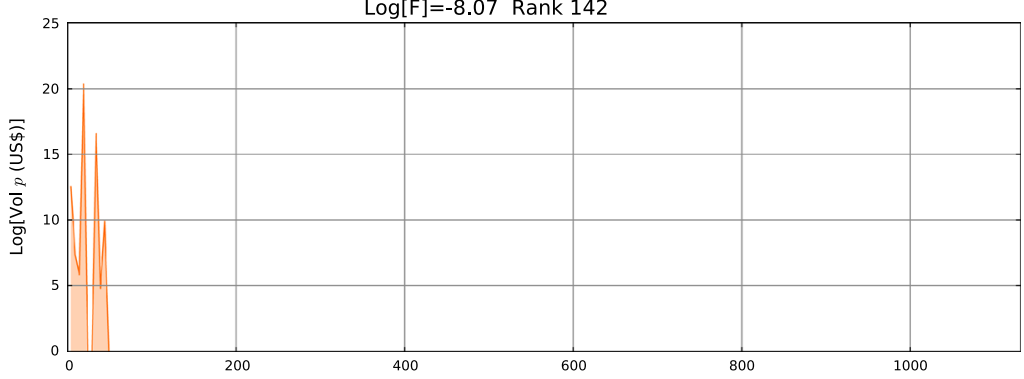

Fig. 1 The countries' spectroscopy shows the distribution of the exported volumes for different products, ordered according to their complexity. One can notice that Netherlands, along with Japan, has not focused on a single class of products; instead, both low complexity and high complexity products are present in their export basket. On the contrary, Nigeria and Qatar, whose economies are based on natural resources, have a poorly diversified export 
low-fitness countries. So in the case of products' complexity a non linear approach must be adopted. Having defined the fitnesses in terms of the complexities, and vice versa, an iterative algorithm has to be constructed: one starts from an initial guess and lets the two maps evolve. In practice, in order to calculate the fitness $F_{c}$ of the country c, and the complexity $Q_{p}$ of the product $\mathrm{p}$, the following set of non linear coupled equations has been proposed (Tacchella et al. 2012):

$$
\begin{aligned}
\tilde{F}_{c}^{(n)} & =\sum_{p} M_{c p} Q_{p}^{(n-1)} \\
\tilde{Q}_{p}^{(n)} & =\frac{1}{\sum_{c} M_{c p} \frac{1}{F_{c}^{(n-1)}}} \\
F_{c}^{(n)} & =\frac{\tilde{F}_{c}^{(n)}}{\left\langle\tilde{F}_{c}^{(n)}\right\rangle_{c}} \\
Q_{p}^{(n)} & =\frac{\tilde{Q}_{p}^{(n)}}{\left\langle\tilde{Q}_{p}^{(n)}\right\rangle_{p}}
\end{aligned}
$$

where the normalization of the intermediate tilded variables is made as a second step by dividing each $\tilde{F}_{c}^{(n)}$ and $\tilde{Q}_{p}^{(n)}$ by the respective averages,

$$
\left\langle\tilde{F}_{c}^{(n)}\right\rangle_{c} \equiv \frac{1}{C} \sum_{c} \tilde{F}_{c}^{(n)} \quad\left\langle\tilde{Q}_{p}^{(n)}\right\rangle_{c} \equiv \frac{1}{P} \sum_{p} \tilde{Q}_{p}^{(n)}
$$

where $C$ and $P$ are the total number of countries and products in the database, respectively, and $n$ is the iteration index. In order to obtain an extensive metrics, which takes into account also the amount of the exports, one can replace the matrix $M_{c p}$ with its weighted counterpart $W_{c p}$, which is defined as $W_{c p}=\frac{q_{c p}}{\sum_{c} q_{c p}}$ where $q_{c p}$ is the export flow out the country $c$ concerning the product $p$. We point out that the case of a monopoly is already included in the algorithm: in this situation the complexity of the product is equal to the exporter's fitness.

The fixed point of these maps has been studied with extensive numerical simulations and it is found to be stable and not depending on the initial conditions. We refer to Cristelli et al. (2013) for an explanation of the features of this approach and for a detailed comparison with the Method of Reflections proposed in Hidalgo and Hausmann (2009). Instead, for a detailed study of the convergence properties of this non linear map, see Pugliese et al. (2014).

\section{Description of data and sectors}

The analyses conducted in this paper are based on the import-export flows as registered in the UN COMTRADE database, processed by BACI (Gaulier and Zignago 2010). This database includes the exports of 200 countries and covers more than 5,000 product categories for a period ranging from 1995 to 2010. The volumes are expressed in the 
same currency (namely, US Dollars, year 2000) so we can compare different countries and different years. There are many possible categorizations for products; we will base our study on the Harmonized System 2007, 4-digit coding, for a total of 1,131 sets of products. After a data cleaning procedure, whose aim is to remove obvious errors in the database records, the number of countries fluctuates between 146 and 148 over the years.

We organize these sets of products taking the sectors which have been recently proposed by the Dutch Government ${ }^{6}$ as a starting point. We are aware that these sectors are in some ways different from the ones usually considered in the economic literature. Since we focus on the Netherlands, we prefer to adopt a partitioning which, in the opinion of the Dutch Government, is more suitable in order to assess their policy. As the original aim of this categorization was different than ours, we expect to have to handle with care the placement of products. The original sectors were 9: Agrifood, Horticulture, High-Tech, Energy, Logistics, Creative Industries, Life Sciences, Chemicals, Water. In the following we will consider only physical products, which does not include services. As a consequence, we will not include Logistics and Water in our analysis. After a first inspection of the database we decide to consider two more sectors: Metals and Other products. Now we briefly describe the sectors, adding some examples for clarity.

1. AgriFood: The integrated agri-food supply chain. This includes products related to food processing, distribution, and retail.

2. Horticulture: All cultivated flowers, vegetables, fruits, plants. There could be a potential overlap with sector 1 in eatable plants, which however turns out to be a small sector. We have included these products in sector 1.

3. High Tech: Mainly electronics and robotics, advanced materials, IT, semiconductors, printing machinery, radars. Cars, trucks and planes are also included.

4. Energy: Products which are directly used to produce energy. Examples include petroleum, natural gas, radioactive elements, wind turbines and solar panels. We do not have electricity among our exports, because only physical products are taken into account. As a counterexample, high tech components suitable for nuclear reactors are put into sector 3 .

5. Creative industries: This sector includes mostly services, for example the TV series Big Brother, with big royalty streams. The physical products within this sector includes only books, movies and related products. The total exported volume, expressed in US dollars, is quite low. We have however considered it for the sake of completeness.

6. Life sciences: Every product that is related to medicine. Examples include pharmaceutical goods, instruments and appliances used in medical, surgical, dental, veterinary or orthopedic sciences, plus the x-ray machinery.

7. Chemicals: Chemical elements, polymers, fertilizers, pneumatic tires, plastic.

8. Metals: Pure metals, without any chemical or industrial process.

9. Other: Everything not included in the previous classification. For example, spores and paper.

\footnotetext{
6 http://www.top-sectoren.nl.
} 
As a first check we compute the total exported volume for each sector and we compare it with the value taken from the respective 2011 policy report for each topsector and the 2012 CBS report (Centraal Bureau voor de Statistiek 2012). We find consistently lower volume values, but this is due to the generality of sectors' definition (in fact, different Dutch institutions come to different results); in particular, the CBS report states that only $40 \%$ of the export is covered by the top sectors. We point out that the total export approximately lines up with the CBS report and that we want to use the top sectors only as a way to allocate the products in logical buckets.

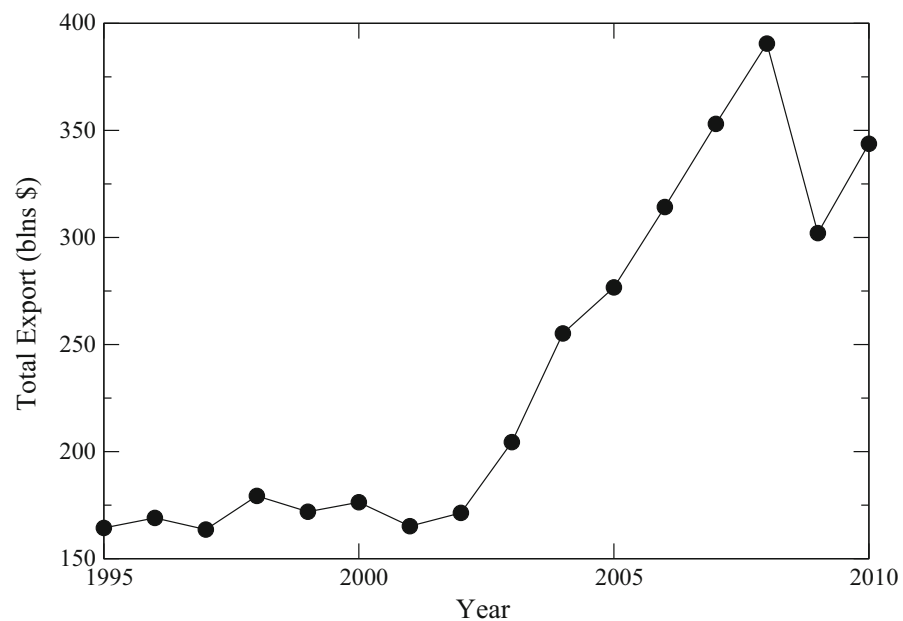

Fig. 2 The total Dutch export, expressed in billion dollars, for the years 1995-2010. One can notice a period of stasis followed by a fast growth from 2003. The evident decline in 2009 is due to the recent crisis

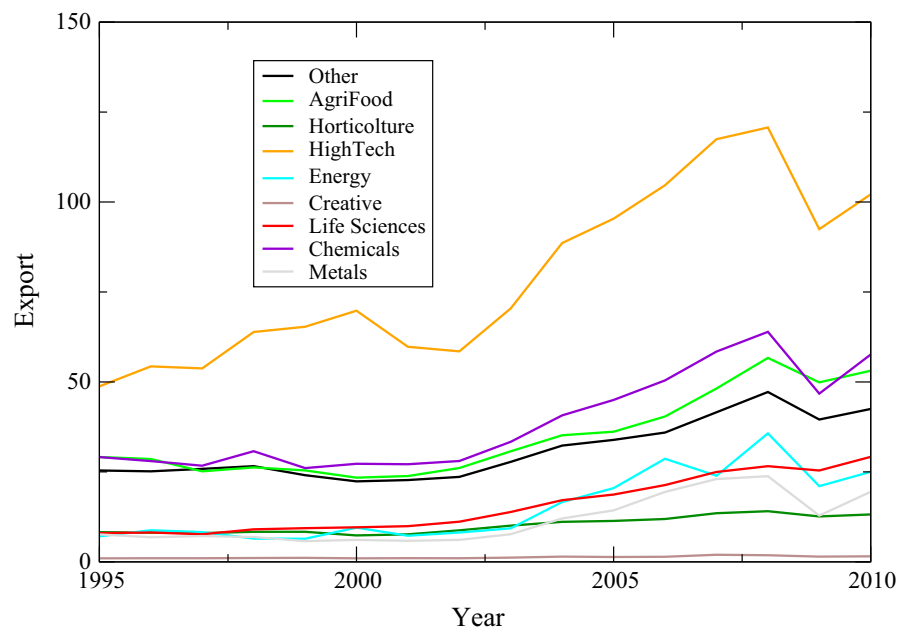

Fig. 3 Dutch exports as a function of time, now divided into sectors. Most of the sectors follow a similar pattern, stressing the presence of a correlation among them. The High-Tech sector is the main contributor of Dutch export. In contrast, the Creative sectors seems to be unimportant with respect to the others 
In Fig. 2 we plot the total exported volume, in billion dollars, as a function of time. Three regimes are present: during the first one, from 1995 to 2002, the export is substantially stable. From 2003 to 2008 volumes rapidly increase until the recent 2009 crisis, which brought Dutch exports to the level of 2006. However, in 2010 a clear sign of recovery is present. This pattern can be approximately noted also if one divides the export basket into sectors, as in Fig. 3, where we plot the time evolution of the volumes for each sector. One can easily see that High Tech represents the main sector of Netherlands' export, covering slightly less than one third of the total volume. On the contrary the Creative sector, which is mostly covered by royalties, which are not included in the products we consider, is practically negligible.

\section{The Netherlands: a novel approach}

\subsection{Top products analysis}

In Fig. 4 we show a synthetic view of the top Dutch exported products, ordered by volume. The columns refer to:

1. a short product description, mainly taken from the Baci website ${ }^{7}$

2. the 4-digit HS2007 code;

3. the product complexity, as defined in Eq. 3;

4. the exported volume, expressed in billion dollars;

5. the Dutch market share, along with the shares of the world seven top competitors;

6. an illustration of the market. Each competitor is represented by a circle, whose center is given by its Fitness, see Eq. 4, and its exported volume. Nations which lie at the top right are the best competitors, because they are high fitness countries which export a lot. The radius of the circle is given by the competitiveness, measured as the ratio between the fitness of the country and the average fitness of the competitors.

One can notice that China, but also USA and Germany (whose code is DEU) are the most "dangerous" competitors for the export of the Netherlands. In fact, often they cover a big part of the same market wherein the Netherlands place its top products. Moreover, these countries share a high level of fitness, which means that they have many different capabilities and, as a consequence, we expect their presence in the market not to fall in the short run.

\subsection{The new description at a glance}

The introduction of a measure of products' complexity gives us the possibility to represent a comprehensive view of the Dutch export in a single graph. In Fig. 5 we plot the exported volumes, expressed in US Dollars, and the average complexity of the sectors for the years 1995 and 2010. The average complexity is calculated weighting each product with its volume:

\footnotetext{
7 http://www.cepii.fr/anglaisgraph/bdd/baci.htm.
} 
Top $\underline{6}$ Products of Netherlands $\underline{2010}$

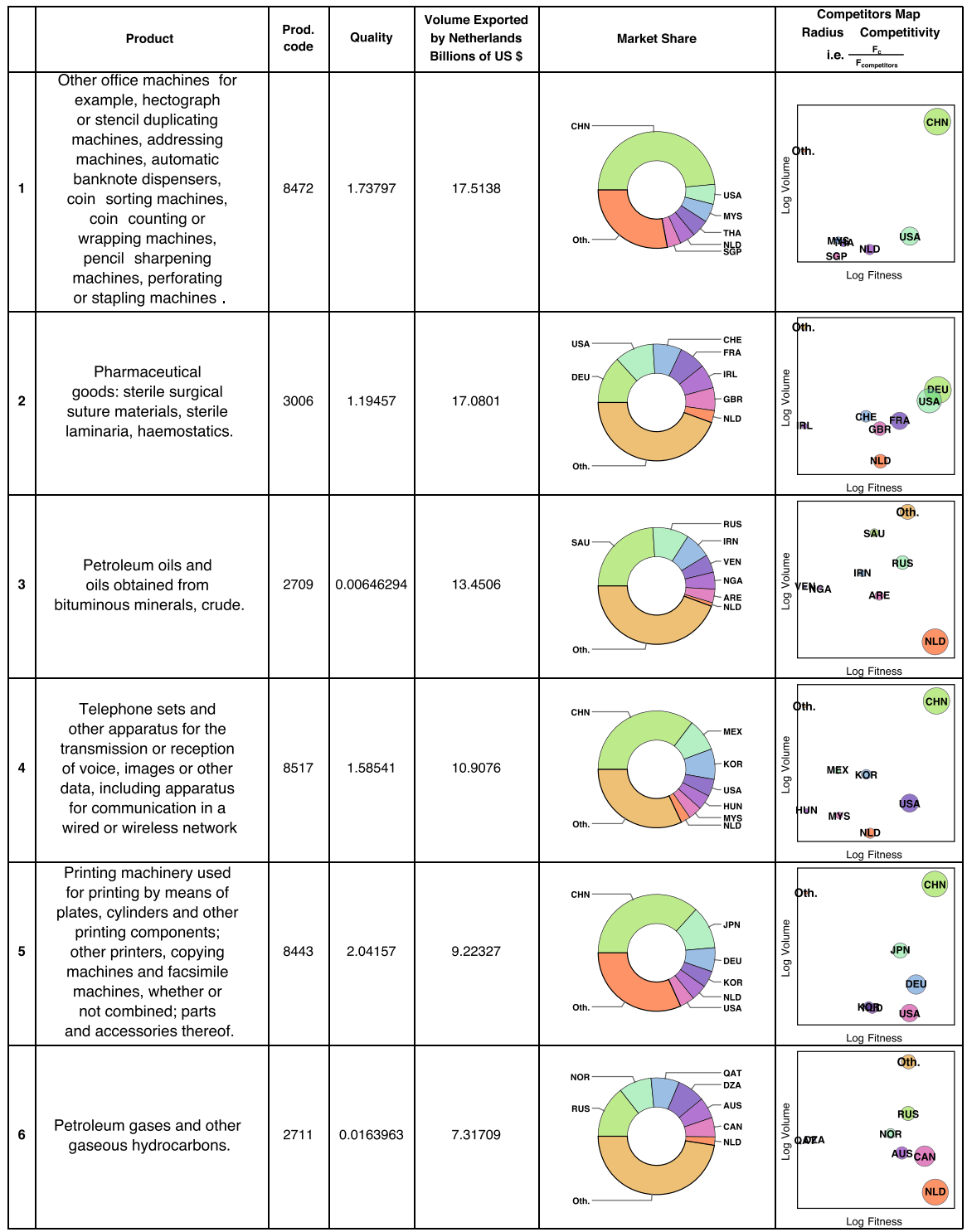

Fig. 4 A synthesis of the main characteristics of the 6 top products of Dutch export. See text for details

$$
\langle Q\rangle_{S}=\frac{1}{V_{\text {tot }}} \sum_{p \in S} V_{p} Q_{p}
$$

where $V_{t o t}=\sum_{p \in S} V_{p}$. With the symbolic expression $\sum_{p \in S}$ we mean that the sum is performed only on those products which belong on one of the sectors previously 

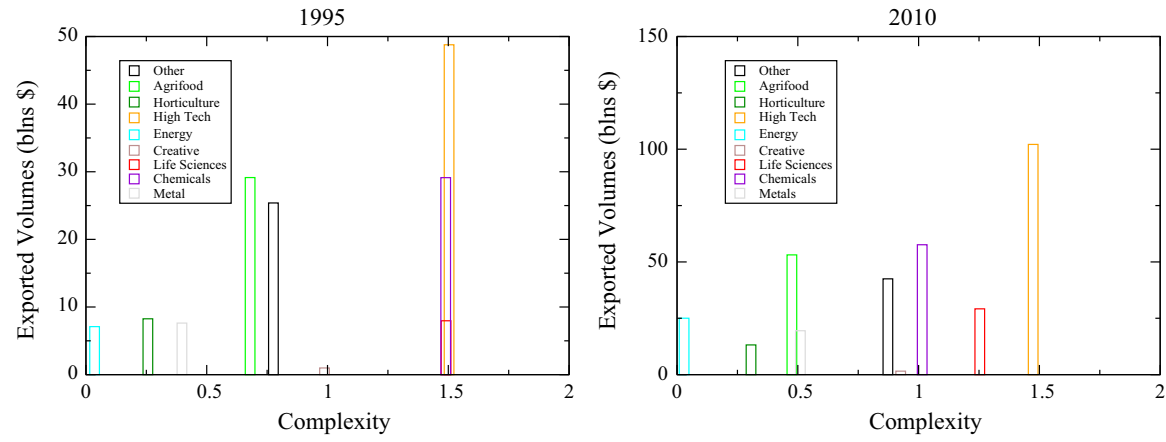

Fig. 5 Dutch exports for the years 1995 and 2010. For each sector the weighted average complexity and the exported volume is plotted

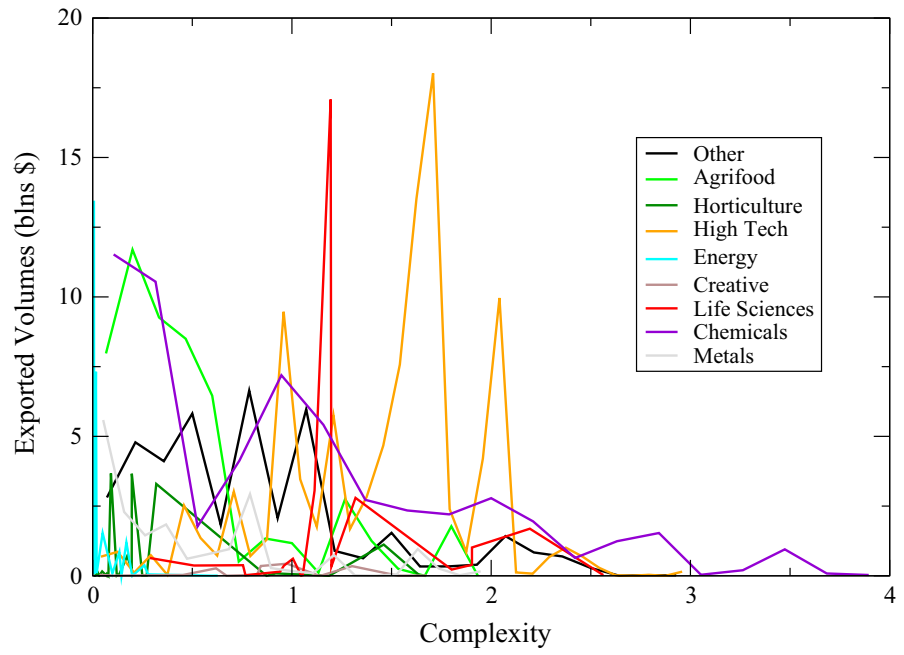

Fig. 6 Volume distributions on the complexity axis for the different sectors for the year 2010

defined; as a consequence, each sector $S$ will be characterized by its weighted average complexity $\langle Q\rangle_{S}$.

One can notice that those sectors which heavily rely on raw materials, as Energy, Horticulture and Metals, share a low complexity, while those sectors which are based on a high level of technology and advanced skills and infrastructures, such as High Tech, Life Sciences and Chemicals, have a high average complexity. A quick comparison between the two years shows that the exports grow for all sectors (note that the $y$ axis has been rescaled). On the contrary, the complexities do not share the same trend: some sectors remain approximatively constant (for example, Energy and High Tech) while others show a remarkable decline, for example Chemicals. We will discuss this aspect in detail in the next Section.

In order to visualize the sector compositions we show in Fig. 6 how the products are distributed along the complexity axis. In other words, we break the columns of 
the previous figures in the respective components, preserving a certain aggregation to improve the readability of the chart. Some sectors, like Energy, are confined to a small interval around the average, while others, like Chemicals, cover a broad range of values. As we will see in the following Section, this fact leads to important consequences for the time evolution of the sectors' complexity.

\subsection{Time evolution}

In this paragraph we study if and how the features of the Netherlands' export changed with time. We start from the simplest analysis, the spectroscopy. In Fig. 7 we show our results for three different years: from top to bottom, 1995, 2002 and 2009. It is evident that the Netherlands' main characteristic, the good diversification of the export, remains unchanged. This is confirmed by a further analysis of the percentiles, which we do not show for reasons of space.

In Fig. 8 we plot the temporal evolution of the sectors' complexity, as defined in Eq. 5. On average, these values are declining. Broadly speaking, this is due to the entrance of low fitness competitors in the market, as expected by the mathematical expression of products' complexity. We note the presence of different patterns in time. For some sectors, for example Agrifood and Chemicals, this trend is constant. The other sectors do not lose complexity in such a consistent way. Given our definition of sectors' complexity as a volume weighted average, we can ask ourselves if this behavior is due to the time evolution of the single products' complexity or to weights rearrangements inside a given sector. In order to answer to questions like these, one usually studies how the average $\left\langle Q^{q_{1}} V^{q_{2}}\right\rangle$ varies with $q_{1}$ and $q_{2}$. Clearly, the exponents regulate the relative weight of the two variables. In particular, for $q_{1}=q_{2}=1$ we recover our definition of weighted average complexity, while for $q_{1}=0$ and $q_{2}=1$ we have, up to normalization constants, the same behavior of Fig. 3. In this case only the volumes count, and we see that in the first half of the considered time interval the export is constant and then it increases. On the contrary, if we consider the case in which $q_{1}=1$ and $q_{2}=0$ we find the average complexity in which each exported product has the same weight. Taking into account the Chemicals sector, we show in Fig. 9 that this quantity is declining in a much less pronounced way. This can mean that either the Netherlands are focused on products which have lost complexity through the years, or that this country has changed its basket from high complexity to low complexity products. In any case, this trending is worrying because it underlines a deterioration of exports' quality, in contrast with the behavior of volumes, which are increasing. 8

In order to confirm this hypothesis quantitatively, we studied the correlation between volumes and complexities inside the Chemicals sector. The Pearson coefficient for 1995 is equal to 0.026 and it grows to 0.126 in 1998, a weak but positive correlation (that is, good products are exported more). Then it starts declining: for the year 2002 we find 0.03 and for $2007-0.048$. For the last year we studied, 2010, the correlation

8 In a similar way we have checked the behavior of the Agrifood sector, finding analogous results. 

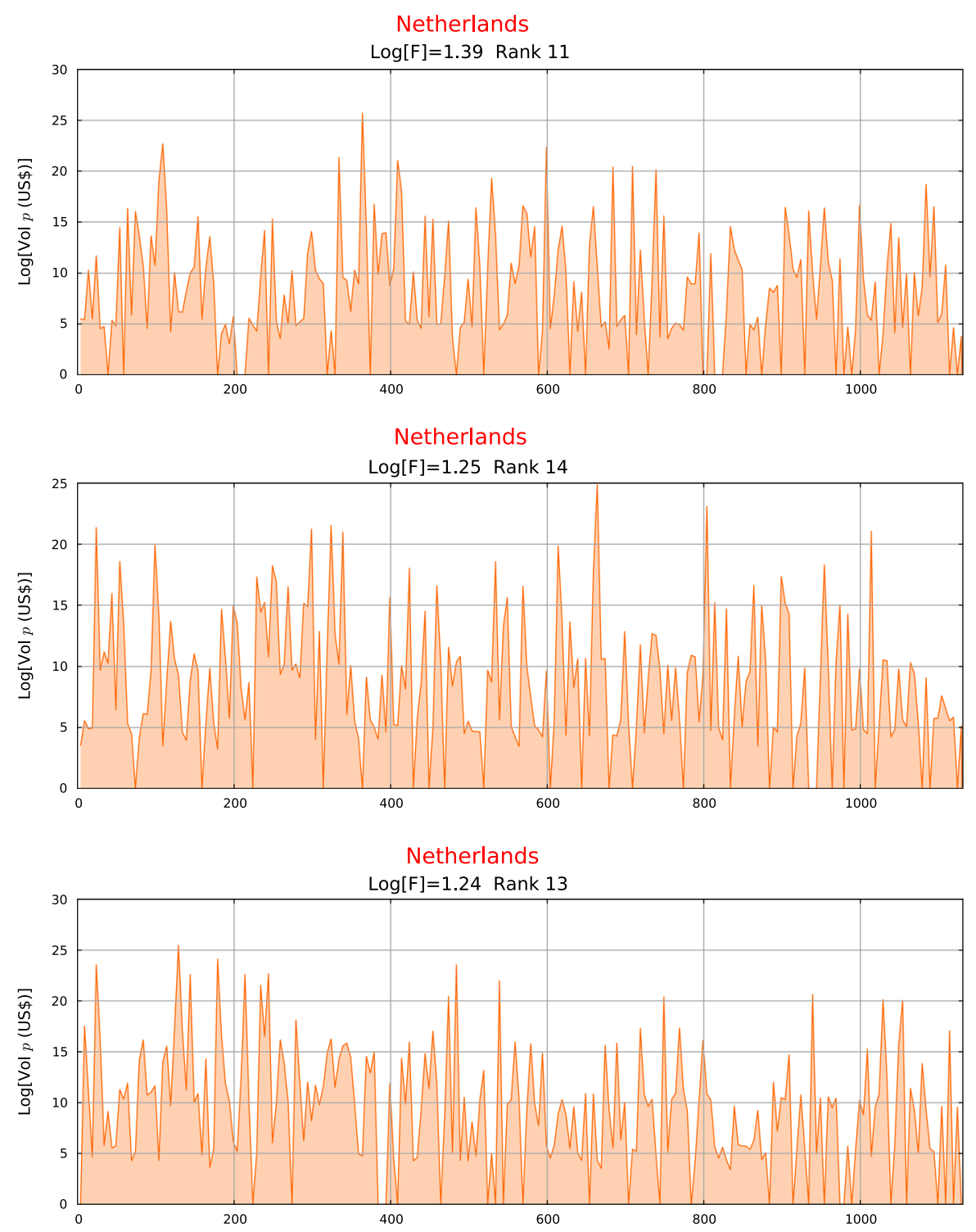

Fig. 7 The spectroscopy of the Netherlands for three different years: from top to bottom, 1995, 2002 and 2009. The country shows an excellent diversification of its products, with no substantial modification over the years

between volumes and complexities decreases to $-0.108 .{ }^{9}$ This behavior evidences that in the last years the good products have lost weight in the Chemicals sector because, on the contrary, a situation in which all the products had lost complexity

\footnotetext{
9 Being the sample size very high (about 200) the correlations are statistically significant for the extreme values. For example, for the 1998 we have a $p$ value of 0.078 for a two tailed test and 0.039 for a one tailed test. Moreover, we observe a declining trend during the years.
} 


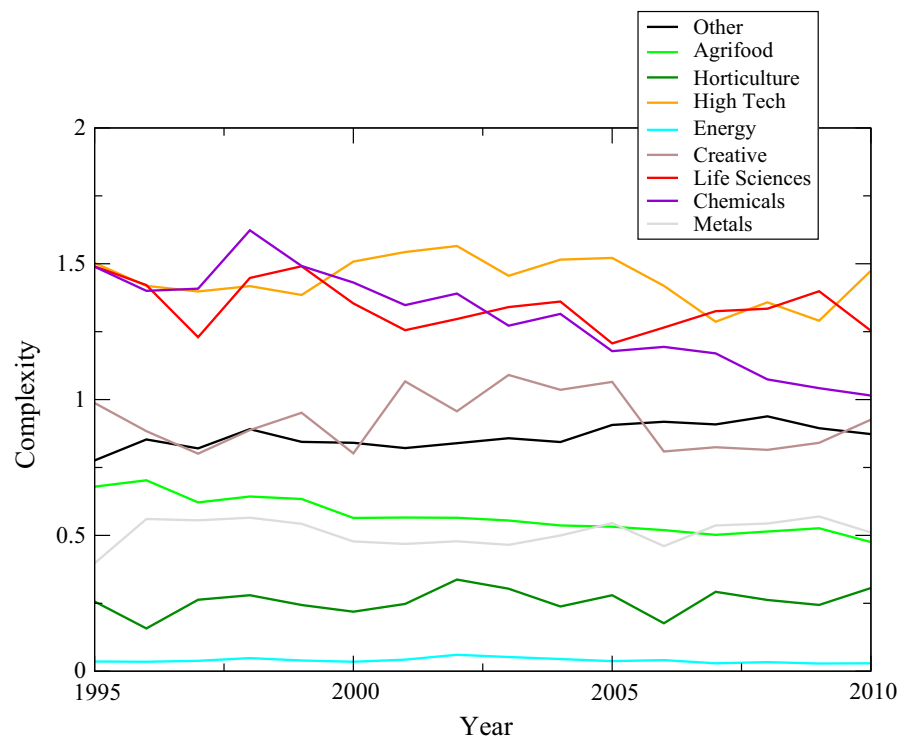

Fig. 8 Sectors' complexity as functions of time. Most of them remain roughly constant, while others, like Chemicals, show a coherent decline over the years

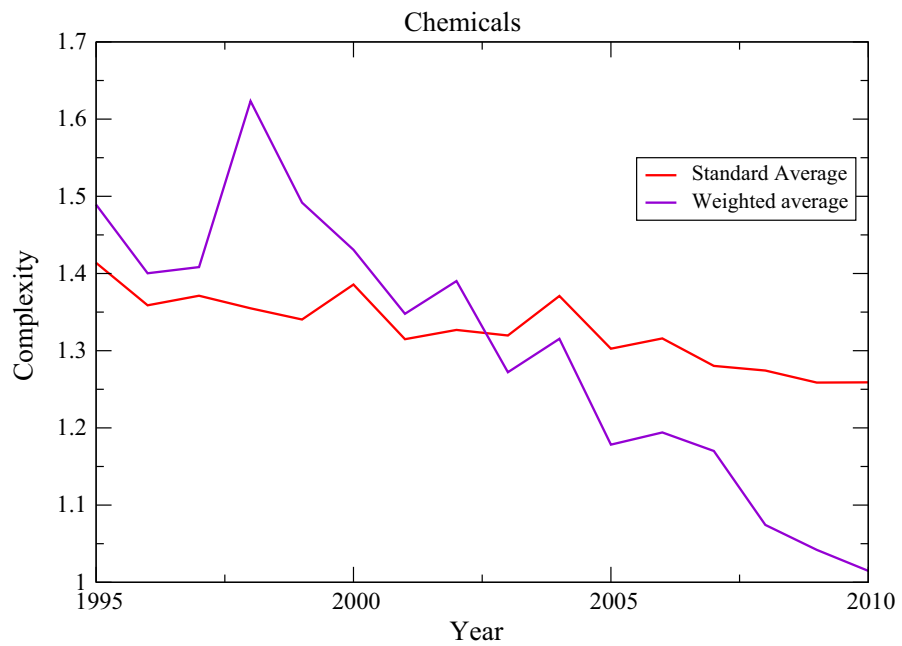

Fig. 9 The time evolution of the simple and weighted average complexity for the Chemicals sector. The latter shows a more remarkable decline

keeping their volumes constant would have led to a constant correlation coefficient. As a consequence, we can state that the complexity loss of this sector has been caused by a weight rearrangement towards lower complexity products over the years.

In order to estimate the growth of the Netherlands we have studied how the other countries evolve, as trajectories in the Fitness-GDP per capita plane, using a recently introduced methodology, which is called Selective Predictability Scheme (SPS) (M. 


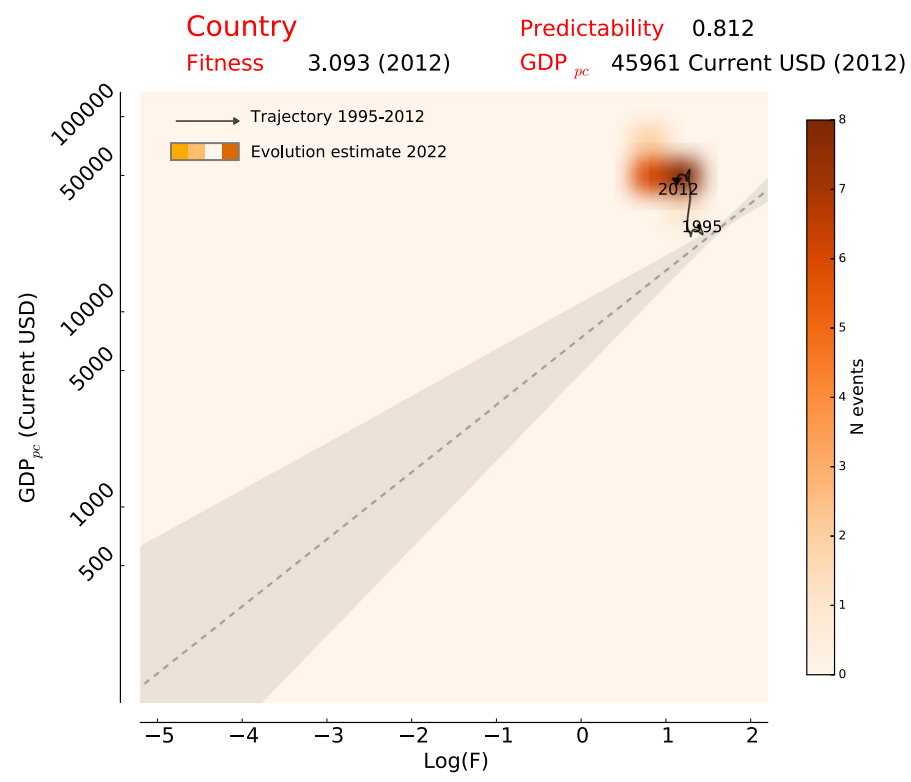

Fig. 10 The results of the SPS analysis predict a substantial stasis of both fitness and GDP per capita in the next 10 years

Cristelli, A. Tacchella and L. Pietronero, Heterogeneous Dynamics of Economic Complexity, Submitted). The basic assumption is that similar initial conditions lead to similar dynamics, resembling the method of analogues, well known in the physics of dynamical systems. The results of our analysis are depicted in Fig. 10. First of all, we have a good predictability. In order to quantify the goodness of our forecasts we use a measure of the future concentration of those points which now are close to the Netherlands. These points, in the past, moved from the area that the Netherlands is crossing to the other areas with a frequency given by the colors. Having the darker areas the same fitness and GDP per capita of 2012, we predict that Netherlands will not considerably grow in the next ten years.

\section{Comparison with other countries and competitiveness}

In Fig. 11 we compare the fitness rankings of various countries with the one of the Netherlands. To compare countries we use the rankings instead of the numerical values because of the algorithm normalization. Indeed, all Western countries are sharing a declining trend of the fitness. This is due to the normalization procedure of the iterations, in which the average fitness is kept constant: as a consequence, if the fitness of the emerging countries increases, the one of the developed countries must decrease. The fitness of the Netherlands is almost constant in the years 1995-2010, revealing that this country not only has preserved the diversification of its basket of export products but also saved it from the emergence of new competitors. Figure 11 gives a rankingsbased comparison which gives, in addition, some insights about other countries. While 
Fig. 11 Time evolution of the fitness for the Netherlands (magenta). Also some Western competitors and the BRIC countries (green) are highlighted (color figure online)

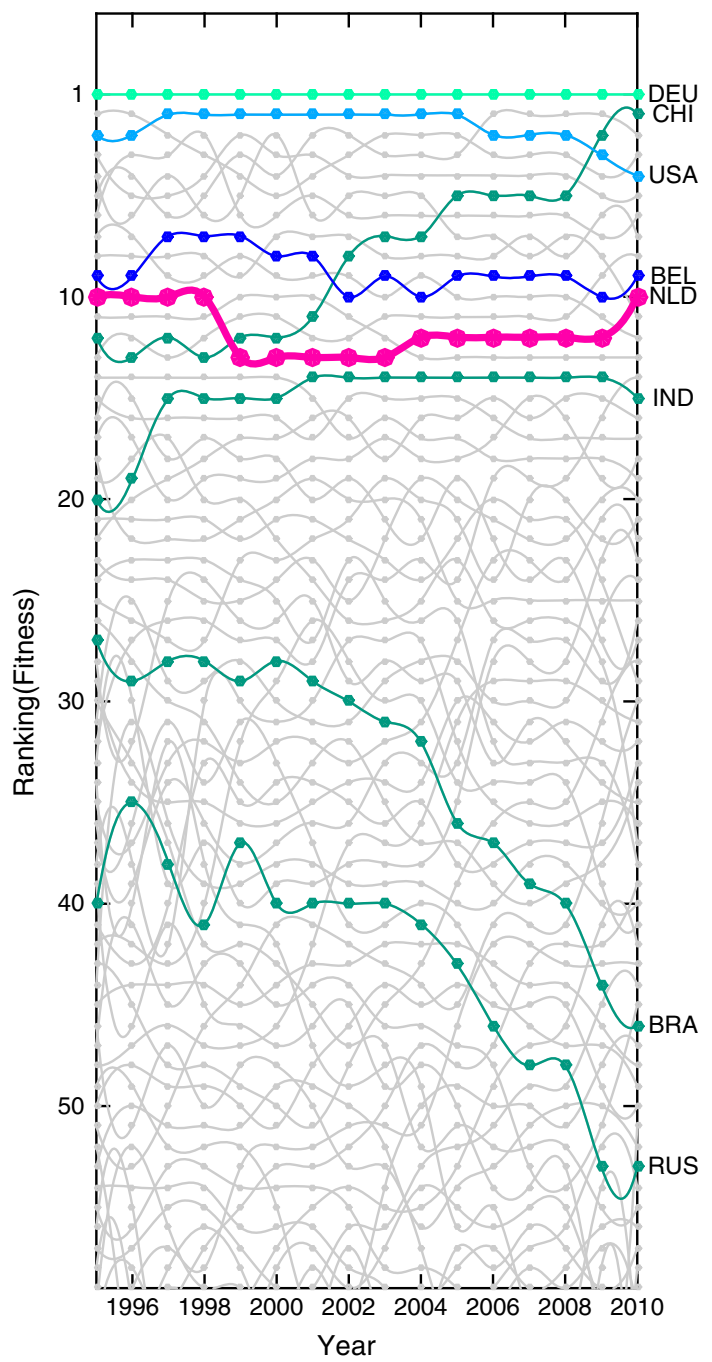

Germany, the Netherlands, and Belgium show a constant behavior, USA experienced a declining trend in the last 5 years. If one considers the patterns of the so called BRIC countries (green lines), it is evident that our methodology splits them in a clear way. Already in 1995 these countries had very different fitness rankings. After an indefinite 5 years phase, in which only India appears to grow, these countries diverge. In particular, while China rises in an impressive way, India remains constant in ranking. On the contrary, Brazil and Russia show a remarkable decline, probably due to an excessive dependence of the export on the primary sector, which has undermined the possible development of other, more complex sectors. Nowadays the presence of different behaviors among the BRICs is a well known fact among the experts; using 


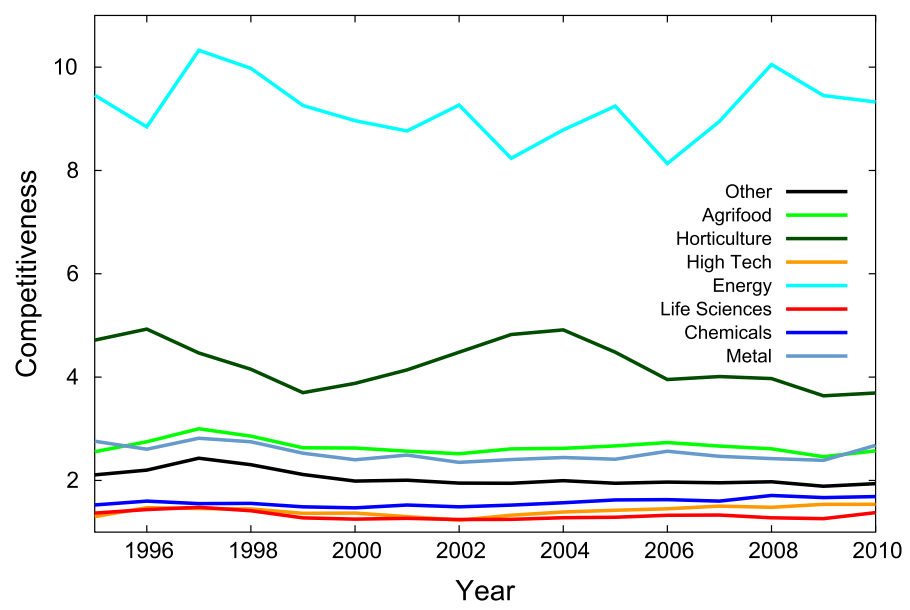

Fig. 12 The time evolution of the sectors' competitiveness. See the text for details

our methodology one can see that this is a result of a trend which started more than a decade ago.

Now we address the issue of quantifying the competitiveness of the Netherlands with respect to other countries, across the different sectors.

Even if, as we have seen in the previous sections, the process by which the complexities are calculated already takes into account the export flows of the other countries, we would like to have a quantification of the importance of the direct competitors of the Netherlands which uses the final results of the iteration procedure. A first proposal is given by the following simple formula. Given the product p exported by the country $\mathrm{c}$, the competitiveness is:

$$
\frac{F_{c}}{\left\langle F_{c}\right\rangle_{\text {competitors }}}
$$

where the denominator is an average over the competitors, that is, those countries whose export of the product $\mathrm{p}$ is above a given threshold. We are aware of the roughness of this approximation: for example, low fitness countries can be very competitive thanks to their low labor cost. However, we believe that a sector without high fitness competitors should be, in principle, richer in opportunity than an full one (Kim and Mauborgne 2005). In Fig. 12 we show how the competitiveness of the sectors evolves with time. The top Dutch sectors from a competitiveness point of view are Energy and Horticulture: the fitness of the competitors is, on average, 4 o 10 times lower than the one of the Netherlands. Despite the low complexity score of these two sectors (see Fig. 8), they have less competitors in the market and so we can state that the Netherlands could focus on these two sector with confidence. At the opposite, the Chemicals sector, which has a high but quickly declining complexity, has a very low competitiveness; we can not recommend to invest only on this sector. 


\section{Conclusion and perspectives}

The aim of this paper is to illustrate the usefulness of some recently introduced instruments to describe and quantify the competitiveness of countries' economy. In particular, the new framework is able to quantify the fitness of a country, that is, its economic, cultural and technological state and the complexity of its exported products, defined in terms of the capabilities needed to prepare or fabricate them. These two quantities are linked by a set of equations which permit a novel measure of these features and, as a consequence, a new point of view from which countries and products can be profitably studied. We applied this methodology to a specific case: the Netherlands. As a first result, from the spectroscopy of the country we can say that the Netherlands show a good diversification, which remains stable through the years. In order to give more precise insights we clustered the products into nine sectors, which we have studied separately. Considering the complexity of the products which make up the sectors, one finds a heterogeneous situation: for example, the Energy sector is primarily made up by low complexity products; on the contrary, the High Tech sector's main contributions come from high complexity exports. Other sectors, like Chemicals, are not focused on a single class of products. From a time evolution perspective we have found that while the growth of the exports can be considered as a common feature of all sectors, for some sectors complexity is declining. We analyzed in detail the case of Chemicals, whose decline has been due to a change of focus towards low complexity products. A first study of sectors' competitiveness suggests that most advantage lies in the Energy and Horticulture sectors, but taking into account that their success is rooted in the highly diverse economic system that supports them. We suggest that our approach, being based on a novel methodology, is a useful complement to the standard approach based on relative export volume ranking.

Being aware of the weight of services in the exports of the Netherlands, we plan to include them a future analysis. Moreover, also imports have been excluded. Given the massive trading position of the Netherlands, we plan to include a discussion of the flow-through volumes and of their added values. For the moment, we believe that our data, which contains the export volumes of all the physical products, can give a sufficiently good view of the global situation of this country. We expect, for example, to find an high degree of diversification also for services.

Acknowledgments We thank Riccardo Di Clemente for providing the artwork for Fig. 11. This work was supported by the European project FET-Open GROWTHCOM (Grant Number 611272) and the Italian PNR project CRISIS-Lab.

Open Access This article is distributed under the terms of the Creative Commons Attribution License which permits any use, distribution, and reproduction in any medium, provided the original author(s) and the source are credited.

\section{References}

Anderson PW (1972) More is different. Science 177(4047):393-396

Balassa B (1965) Trade liberalisation and "revealed" comparative advantage. Manch Sch 33(2):99-123. Wiley Online Library 
Barro RJ, Sala-i Martin X (2004) Economic growth. MIT Press, Cambridge

Caldarelli G, Cristelli M, Gabrielli A, Pietronero L, Scala A, Tacchella A (2012) A network analysis of countries' export flows: firm grounds for the building blocks of the economy. PloS One 7(10):e47278

Centraal Bureau voor de Statistiek (2012) Monitor Topsectoren - Uitkomsten eerste meting, http://tinyurl. com/lhwuzjn (in Dutch). Accessed 7 Feb 2014. Technical report

Cristelli M, Gabrielli A, Tacchella A, Caldarelli G, Pietronero L (2013) Measuring the intangibles: a metrics for the economic complexity of countries and products. PLoS One 8:e70726

Dixit AK, Stiglitz JE (1977) Monopolistic competition and optimum product diversity. Am Econ Rev 67:297-308

Dosi G, Pavitt K, Soete LL (1990) The economics of technical change and international trade. LEM Book Series

Flam H, Flanders M (1991) Heckscher-Ohlin trade theory. MIT Press, Cambridge

Gaulier G, Zignago S (2010) Baci: international trade database at the product-level. Centre d'Etudes Prospectives et d'Informations Internationales http://www.cepii.fr/anglaisgraph/workpap/pdf/2010/ wp2010-23

Grossman G, Helpman E (1991) Quality ladders in the theory of growth. Rev Econ Stud 58:43-61

Hausmann R, Rodrick D (2003) Economic development as self-discovery. J Dev Econ 72:603-633

Hausmann R, Hidalgo CA, Bustos A, Coscia M, Chung S, Jimenez J, Simoes A, Yildirim M (2011) The atlas of economic complexity. Puritan Press, Cambridge

Hidalgo CA, Klinger B, Barabási AL, Hausmann R (2007) The product space conditions the development of nations. Science 317(5837):482-487

Hidalgo C, Hausmann R (2009) The building blocks of economic complexity. Proc Natl Acad Sci USA 106:10570-10575

Hidalgo C, Hausmann R (2010) Country diversification, product ubiquity, and economic divergence. John F. Kennedy School of Government, Harvard University

Jones CI (1995) R\&D-based models of economic growth. J Polit Econ 103(4):759

Kim WC, Mauborgne R (2005) Blue ocean strategy: how to create uncontested market space and make competition irrelevant. Harvard Business Press, Cambridge

Krugman P (1979) Increasing returns, monopolistic competition, and international trade. J Int Econ 9(4):469-479

Krugman P, Wells R (2013) Macroeconomics. Worth Publishers, New York

Lall S, Weiss J, Zhang J (2006) The sophistication of exports: a new trade measure. World Dev 34(2):222237

Lall S (2006) The technological structure and performance of developing country manufactured exports, 1985-1998. Oxf Dev Stud 28:337-369

Pugliese E, Zaccaria A, Pietronero L (2014) On the convergence of the fitness-complexity algorithm arXiv:1410.0249

Ricardo D (1817) On the principles of political economy and taxation. John Murray

Romer P (1990) Endogenous technological change. J Polit Econ 98:71-102

Smith A (1776) The wealth of nations. Strahan and Cadell

Tacchella A, Cristelli M, Caldarelli G, Gabrielli A, Pietronero L (2012) A new metrics for countries' fitness and products' complexity. Sci Rep 2:723

Tacchella A, Cristelli M, Caldarelli G, Gabrielli A, Pietronero L (2013) Economic complexity: conceptual grounding of a new metrics for global competitiveness. J Econ Dyn Control 37(8):1683-1691

Zaccaria A, Cristelli M, Tacchella A, Pietronero L (2014) How the taxonomy of products drives the economic development of countries. PLoS One 9(12):e113770 